\title{
EXPERIMENTAL DETERMINATION OF THE ZERO POWER TRANSFER FUNCTION OF THE AKR-2
}

\author{
Sebastian Hübner ${ }^{1}$, Alexander Knospe ${ }^{1}$, \\ Marco Viebach ${ }^{1}$, Carsten Lange ${ }^{1}$, and Antonio Hurtado ${ }^{1}$ \\ ${ }^{1}$ Technische Universität Dresden (TU Dresden) \\ 01062 Dresden
}

sebastian.huebner@tu-dresden.de, alexander.knospe@tu-dresden.de, marco.viebach@tu-dresden.de, carsten.lange@tu-dresden.de, antonio.hurtado@tu-dresden.de

\begin{abstract}
The transfer function is a basic characteristic of every nuclear reactor. It describes how a perturbation at a given place and time influences the neutron flux. In case of a known perturbation, the determination of characteristic reactor parameters is possible. The present paper shows an experimental method to determine the gain of the zero-power reactor transfer function (ZPTF) of the AKR-2 reactor at TU Dresden and the comparison to the theoretical shape of the ZPTF derived from kinetic parameters simulated with MCNP. For the experiments, a high-precision linear motor axis is used to insert an oscillating perturbation acting at frequencies smaller than the lower bound of the plateau region of the ZPTF. For higher frequencies, a rotating absorber is used. This device emulates an absorber of variable strength. The reactor response is detected with a He-3 counter.

The data evaluation shows good agreement between measured and corresponding theoretical values of the gain of the ZPTF.
\end{abstract}

KEYWORDS: transfer function, zero-power reactor, noise analysis, training reactor, MCNP simulation

\section{INTRODUCTION}

The transfer function is a basic characteristic of every nuclear reactor. It describes how a perturbation at a given place and time influences the reactor's state variables [1]. The concept of a transfer function is reliably applicable as long as the system's behavior can be approximated by a linear time-invariant dynamical system. The knowledge of the transfer function may allow determining the type of the perturbation from the measurements of the induced fluctuations of the neutron flux or, in case the perturbation is known, the determination of characteristic reactor parameters [2]. The determination of the reactor transfer functions has been performed since the early days of reactor research [3]. Nevertheless, in current safety research, the inversion of the transfer function as a method of incident detection is of particular interest [4].

In this context, the gain of the transfer function of the AKR-2 reactor at TU Dresden is experimentally determined by use of a vibrating absorber and an absorber of variable strength. Since thermal feedback effects in the AKR-2 core can be neglected during normal operation, the AKR-2 can be assumed to be a zero-power reactor. Moreover, the dynamical behavior of the AKR-2 can be described by the point-kinetic approximation. In such conditions, the transfer function is determined by the zero power transfer function (ZPTF), with the applied perturbation converted into its reactivity effect. For the experiments, a highprecision linear motor axis is used to insert an oscillating perturbation acting at frequencies smaller than 
the lower bound of the plateau region of the ZPTF. For higher frequencies, a rotating absorber is used. This device emulates an absorber of variable strength. The reactor response is detected with a He-3 counter placed inside the reactor. Using this setup, the gain of the ZPTF of the AKR-2 is measured through the analysis of the input signals (movement of the absorber) and the output signals (induced fluctuations in neutron flux). For an assessment of the measured ZPTF, it is compared with the theoretical shape of the ZPTF derived from kinetic parameters that were simulated with an MCNP model of the reactor.

Based on the knowledge of the ZPTF, the investigation of neutron flux fluctuations using the same experimental setup will be possible in the future. Conceivable research fields could be the generation of high-precision data with a high level of fidelity for the validation of computer codes, inducing one or more perturbations. Additionally, a direct evaluation of unknown cross sections of different materials with the pile-oscillator method would be possible.

\section{THEORY OF ZERO POWER TRANSFER FUNCTION}

The transfer function $G(s)$ describes the transfer behavior of a system, which reacts to an input signal replying with a corresponding output signal in the frequency domain. For a nuclear reactor, the input signal is a reactivity deviation that leads to a local or global deviation of the reactor's state variables. As for a zero power reactor thermal and other feedback effects are neglected. Input and output are coupled just with the ZPTF [5]. The input signal is a deviation of the reactivity $(\rho)$, which leads to a change in the neutron flux $(\Phi)$. This transfer is coupled via the transfer function $(G)$.

Mathematical description of the ZPTF is derived from the point kinetic approximation. The derivation is widely described in many publications [1,2]. The following formulation is the transfer function in frequency domain:

$$
G(s)=\frac{n_{0}}{l s+\sum \beta_{i}-\rho_{0}-\sum_{i} \frac{\beta_{i} \lambda_{i}}{s+\lambda_{i}}} .
$$

Where $\beta$ is the fraction of delayed neutrons, $\lambda$ is the decay constant of the precursors, both for the $i$-th group of precursors, and $l$ the generation time of the prompt neutrons. The initial neutron number $\left(n_{0}\right)$ is commonly used as normalization factor. The gain of the ZPTF is represented by the absolute value of $G(s)$. For a sinusoidal perturbation is $s=i \omega$.

\section{DESCRIPTION OF THE EXPERIMENTAL SETUP}

The experiments to determine the ZPTF were carried out at the AKR-2 reactor with experimental equipment that was developed in the context of the European project CORTEX [4] for the validation of simulation tools. The low frequency range $(0.05 \mathrm{~Hz}$ to $1 \mathrm{~Hz})$ was examined with a vibrating absorber and the higher frequencies $(0.5 \mathrm{~Hz}$ to $12 \mathrm{~Hz})$ with an absorber of variable strength. In the following, the reactor is briefly introduced, as well as the two different experimental setups.

\subsection{The AKR-2 reactor}

The AKR-2 reactor [6], located at TU Dresden, is a thermal, zero-power reactor with an allowed maximum thermal power of $2 \mathrm{~W}$. It is used mainly for training and teaching purposes, and additionally for research. The core has cylindrical shape with a diameter of $250 \mathrm{~mm}$ and a height of $275 \mathrm{~mm}$. The disk-shaped fuel elements consist of a homogeneous dispersion of polyethylene moderator and uranium oxide, which is enriched to $19.8 \%$. For security reasons the core is separable, whereas the lower half is movable. For the basic start-up procedure, the core halves are brought together. The operation is controlled via three control and safety rods installed next to the fuel zone, consisting of cadmium sheets on polyethylene blocks. A graphite reflector with approx. $32 \mathrm{~cm}$ thickness surrounds the core. The biological shield consists of two 
cylindrical walls of $15 \mathrm{~cm}$ and $58 \mathrm{~cm}$ thickness, made of paraffin and heavy concrete. The reactor is accessible through seven horizontal and two vertical experimental channels. The setup of the AKR-2 is illustrated in Figure 1.

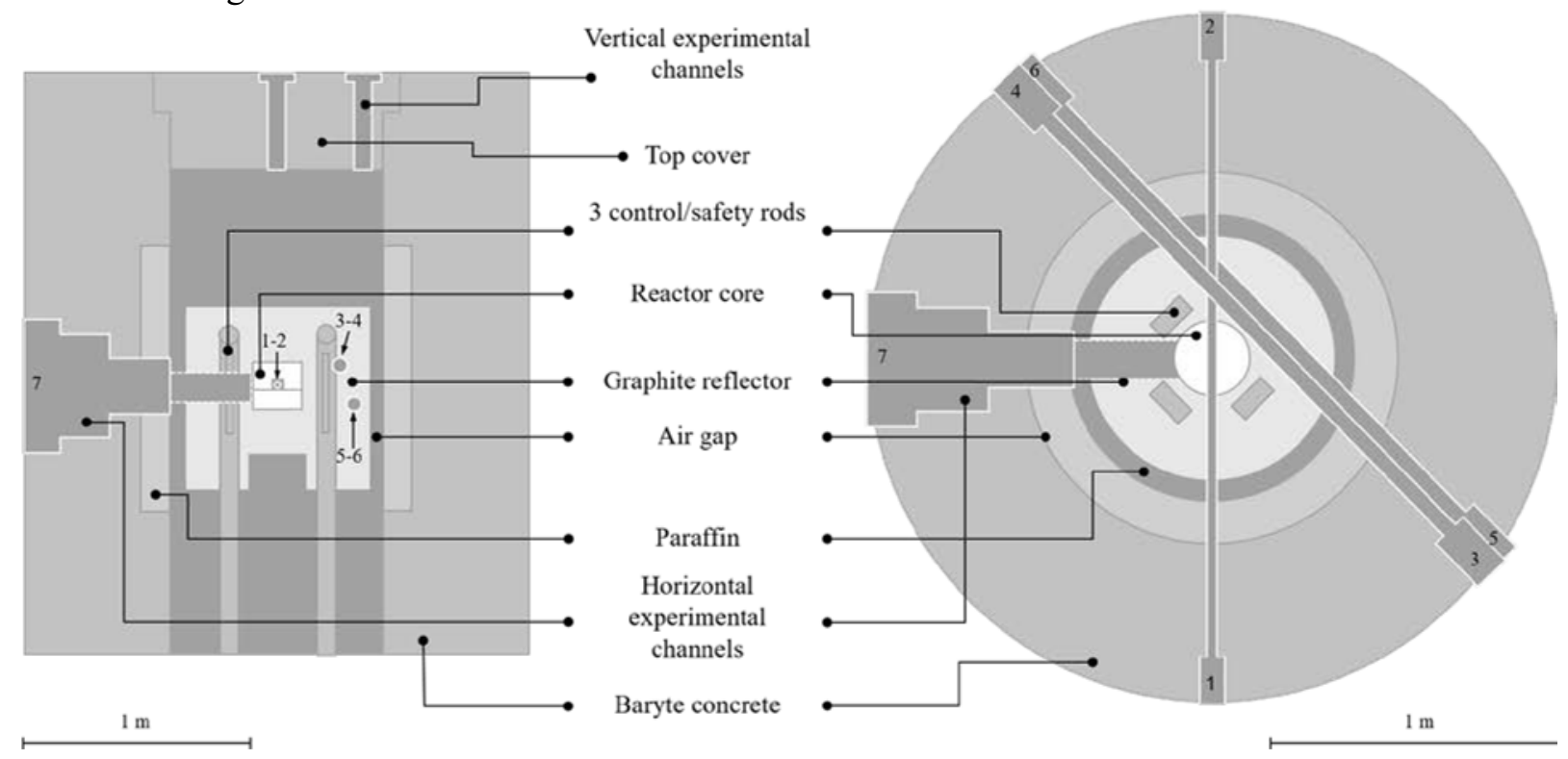

Figure 1. Vertical and horizontal cross section of the AKR-2.

\subsection{Vibrating absorber}

The vibrating absorber triggers a repetitive perturbation in the reactor. For the AKR-2 this is realized with the help of a linear motor axis that moves a shaft containing a cadmium absorber within the experimental channel 1-2. The absorber is a cadmium cylinder with a diameter of $12.7 \mathrm{~mm}$ and a height of $1.016 \mathrm{~mm}$. It consist of $99.9656 \%$ natural cadmium and minor impurities of other metals. The setup can be seen in Figure $2 \mathrm{a}$ ). The linear motor axis can effect different motion profiles with a maximum amplitude of $15.5 \mathrm{~cm}$ with a positioning accuracy of $10 \mu \mathrm{m}$ and a repositioning accuracy of $10 \mu \mathrm{m}$. Because of safety limitations of the driver software of the axis, a repeatable motion profile can only be guaranteed up to a frequency of $1 \mathrm{~Hz}$. Frequencies lower than realized in the shown experiments are not possible due to the reactor response, which would exceed the reactor's safety (power) margins. For the experiments, a rectangular motion shape was chosen with an amplitude of $2 \mathrm{~cm}$ (between $7 \mathrm{~cm}$ and $11 \mathrm{~cm}$ from core center). This assures the movement of the absorber inside a zone with a linear decrease of the neutron flux, resulting in a linear transfer of the reactivity perturbation. The induced reactivity over the absorber position is shown in Figure $2 \mathrm{~b}$ ). The reactivity difference between the maximum and the minimum position of the absorber is $0.055 \$$. The reactivity influence over the movement path was determined via compensationmethod with help of one the control and safety rods. The movement of the axis is controlled and recorded by the programmable logic controller of the linear motor axis via an absolute position encoder.

\subsection{Absorber of variable strength}

The absorber of variable strength inserts a local reactivity perturbation. For the AKR-2, this is realized with the help of an absorber, rotating in the experimental channel 3-4 and driven by a stepper motor. The absorber is a bent rectangular cadmium sheet with size of $25 \mathrm{~cm} \times 2 \mathrm{~cm} \times 0.02 \mathrm{~cm}$ with a bending and rotation radius of $2.98 \mathrm{~cm}$. The largest dimension is parallel to the experimental channel. The material of the absorber is natural cadmium with unknown impurities. 
a)

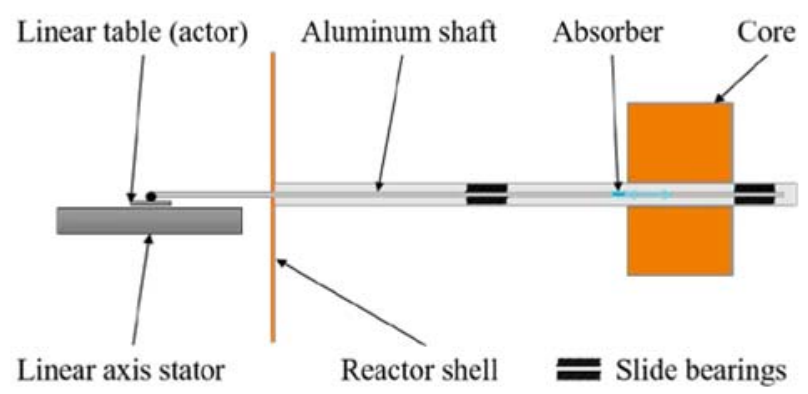

b)

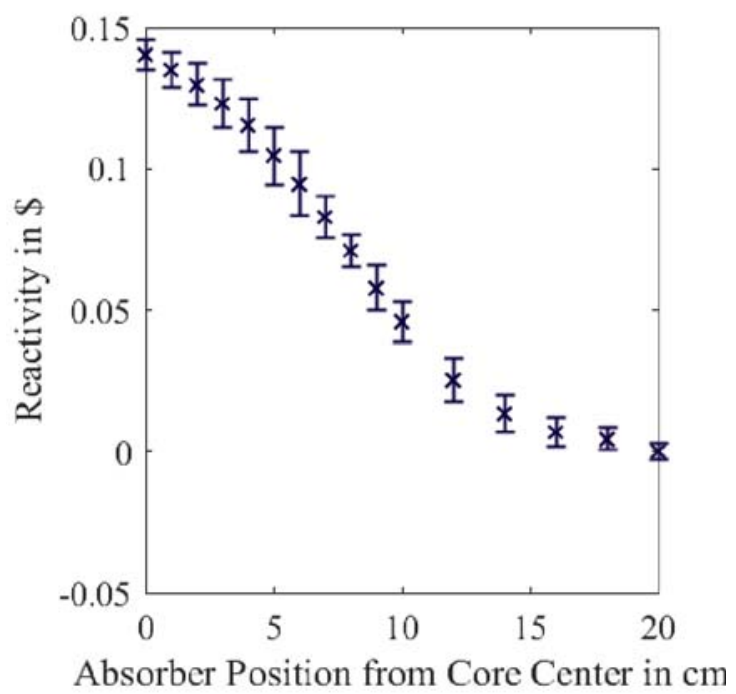

Figure 2. a) Schematic and b) Reactivity influence of the vibrating absorber.

The stepper motor induces a motion profile with a constant angular velocity. Frequencies range from $1 \mathrm{~Hz}$ to $12 \mathrm{~Hz}$. The perturbation is started in the position closest to the ground level (as can be seen in Figure 3 a). This position is referenced as angle $0^{\circ}$. The movement starts towards the reactor core. The difference of the minimum and maximum reactivity of a whole repetition of the absorber is $0.011 \$$. The reactivity influence was determined in the same way as the one for the other absorber type. The reactivity over the angle is illustrated in Figure $3 \mathrm{~b}$ ). In the present case, it was not possible to track the movement of the stepper-motor. This led to the assumption of a continuous movement with constant angular velocity, which was checked by a time control for the lower frequencies and a sound noise analysis for the higher frequencies.

a)

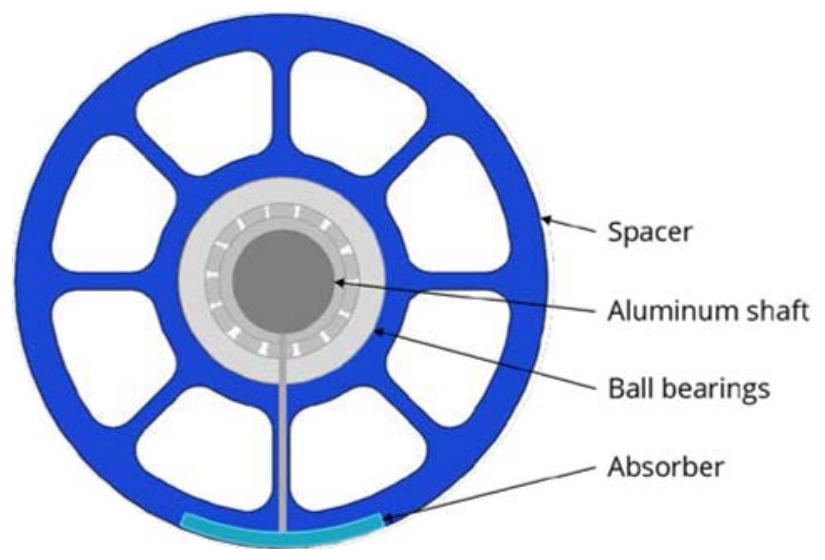

b)

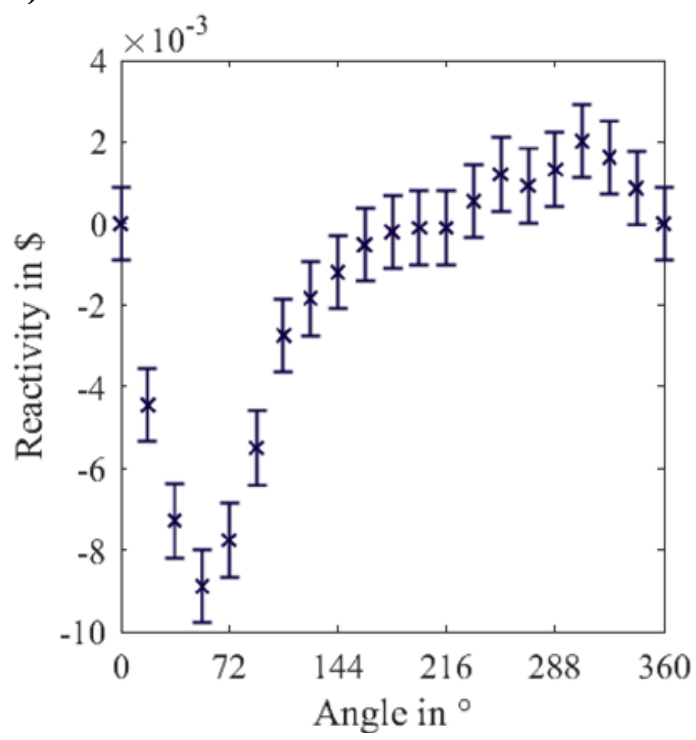

Figure 3. a) Schematic and b) Reactivity influence of the absorber of variable strength. 


\subsection{Measurement setup with detector position}

The used detector was a He- 3 counter in pulse mode operation. The volume of the effective, helium-filled zone was determined via computed tomography: $V=12.441 \mathrm{~cm}^{3}\left(+0.638 \mathrm{~cm}^{3} /-0.181 \mathrm{~cm}^{3}\right)$. The signal was amplified and then recorded with an ORTEC multi-channel-scaler. The position of the detector and of the perturbations during the experiments is shown in Figure 4.

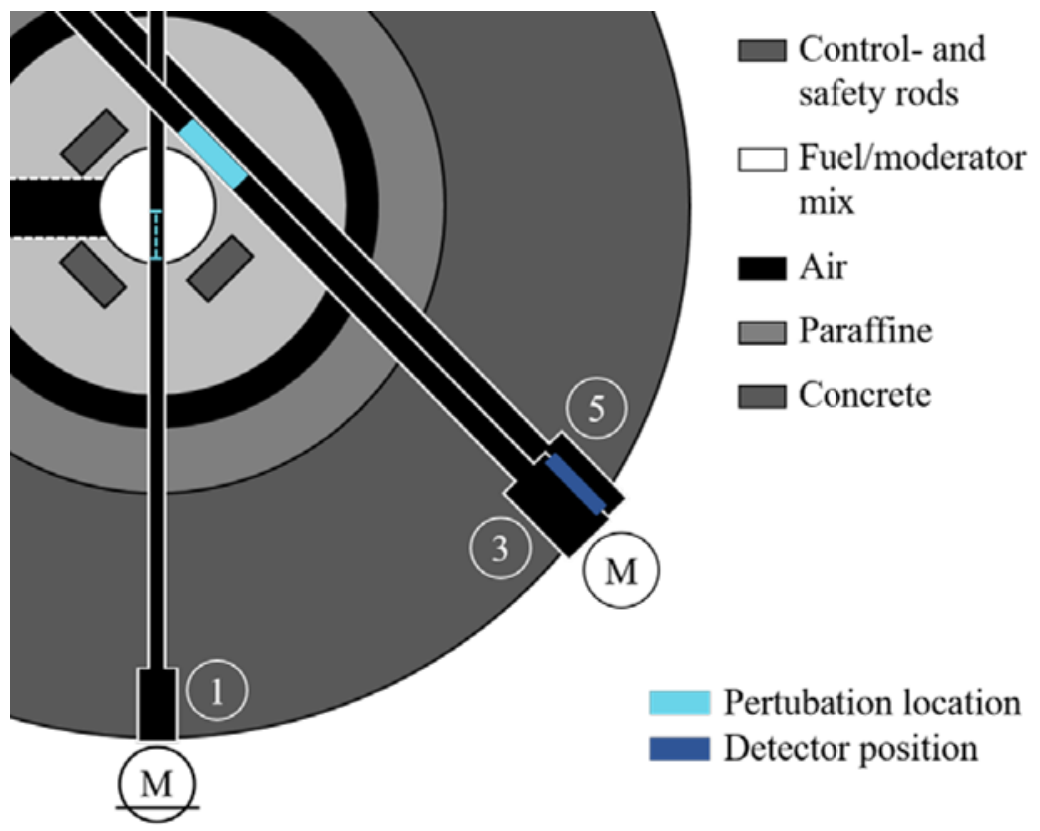

Figure 4. Partial cross section of the AKR-2 with the experimental setup:

Cross section mesh-up of three cross sections of the AKR-2 $(-8 \mathrm{~cm}, 0 \mathrm{~cm},+8 \mathrm{~cm}$ in vertical direction, regarding the core center). The experimental channels $1,3,5$ can be seen as well as the location of the detector (dark blue) and the perturbation locations (light blue). The vibrating absorber is located in channel 1, the absorber of variable strength in channel 3 and the detector in channel 5.

\section{MEASUREMENTS AND DATA ANALYSIS}

The measurements were performed at four different days with a displayed power for each measurement of $1.5 \mathrm{~W}$. The chosen perturbation frequencies range from $0.01 \mathrm{~Hz}$ and $12 \mathrm{~Hz}$. For an assessment of the uncertainties of the measurement, experiments were repeated for some of the chosen frequencies.

The input signals for the analysis of the transfer function are the reactivity influences of the absorbers. They are obtained by adjusting the recorded motion profiles with the measured reactivity influence information in reference to the absorber positions (compare Figure $2 b$ ) and Figure $3 b$ ).

The output signals are the recorded neutron counts. With limited time for each measurement, it was difficult to always obtain a perfect critical state before starting the recording. To control whether the critical state is reached, the decay of the majority of the precursors has to be waited for after a movement of the control and safety rods. In case of a decrease or an increase of the reactor power, the reactor state has to be adjusted recursively. This would have exceeded the available measurement time. To overcome this challenge, for each measurement, the baseline of the recorded output data was fitted with a polynomial of second order. Subsequently, the fit curve was subtracted from the signal. The fits represent the increase or decrease of the power due the influence of the precursors with the larger half-life. The concentration of these precursors are not influenced by the relatively high frequencies of the current experiment. The applicability of this method on the decay of precursors was shown for pile-oscillator experiments [7]. 
a)

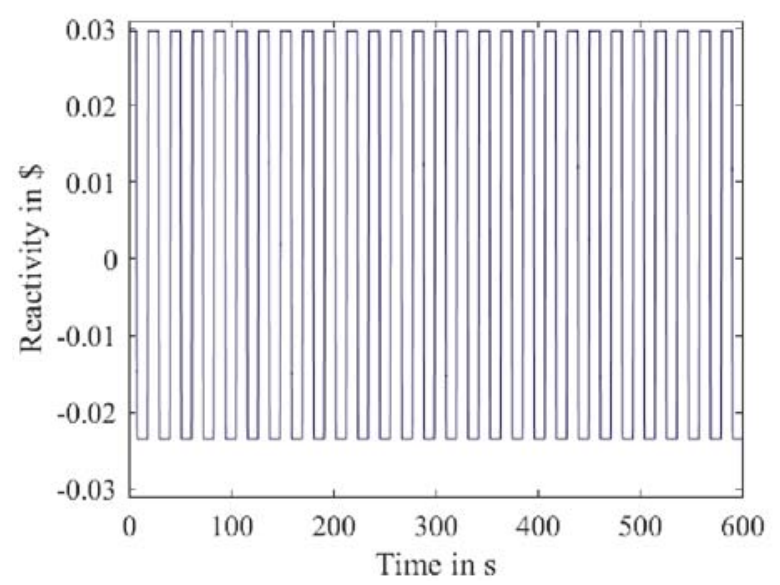

c)

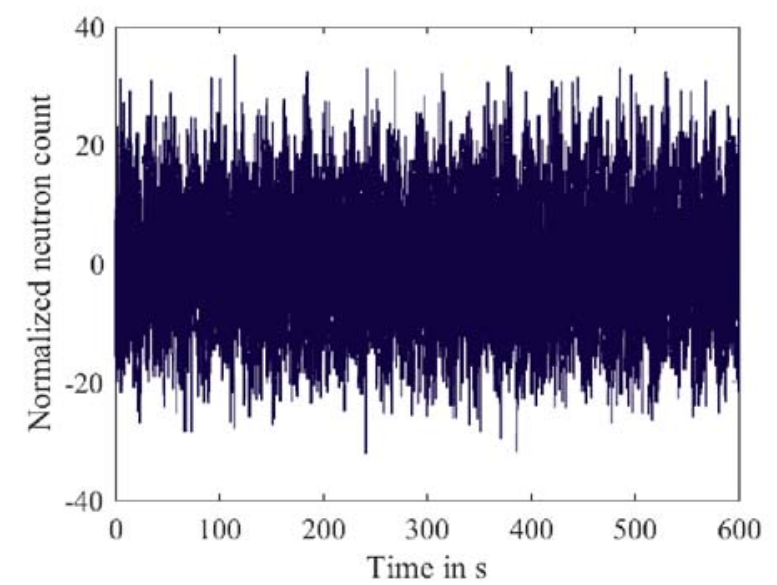

b)

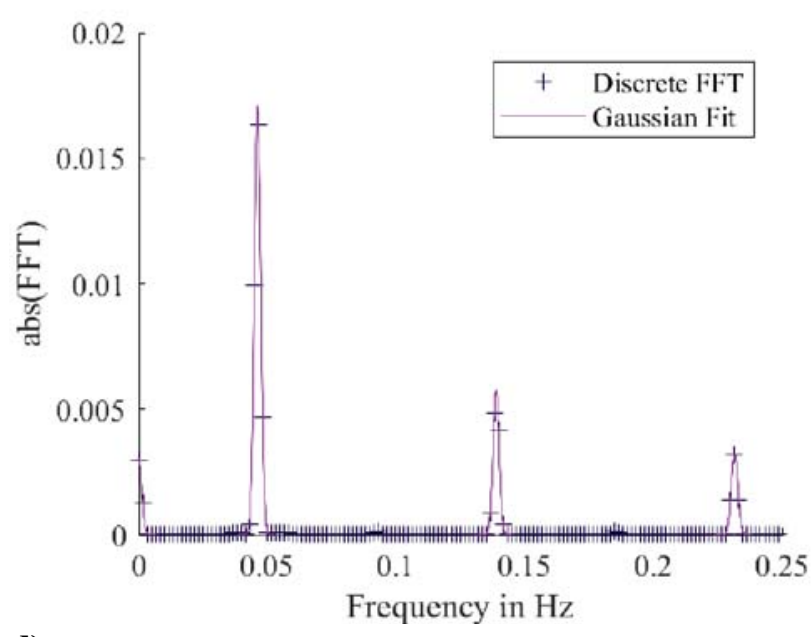

d)

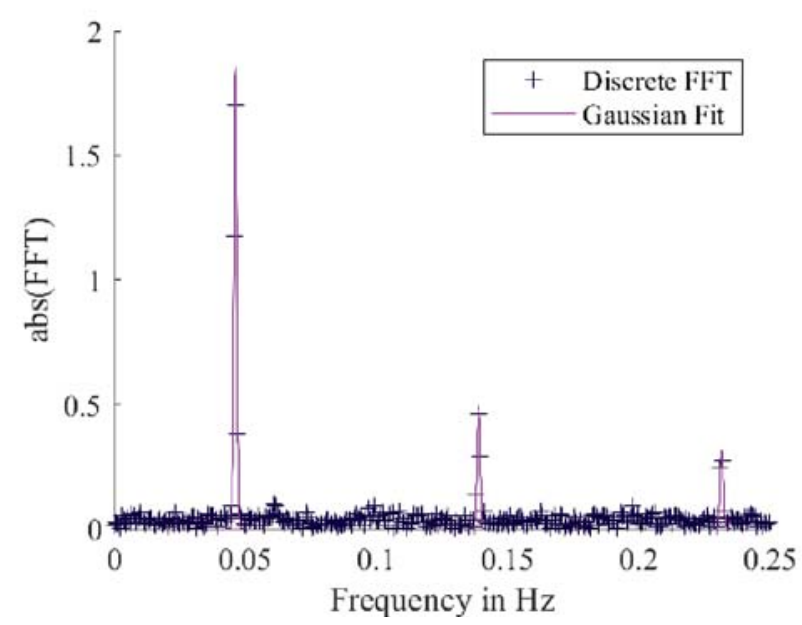

Figure 5. a)-d) Signal example for measurement with vibrating absorber with perturbation frequency of $0.05 \mathrm{~Hz}$ :

Input signal (a) with corresponding discrete FFT with Gaussian peak fits (b) and output signal with corresponding (c) discrete FFT with Gaussian peak fits (d).

A Fast Fourier Transform (FFT) transfered both, input and output data separately, to the frequency domain. The FFT was performed using a periodic Hamming window [8]. The main peaks for every frequency domain data set were identified and fitted with a Gaussian peak function in an automated manner. The peak heights in frequency domain are an equivalent representation of the amplitude of the signals of the input and of the output, respectively. This assumption is reliable, as the peak shapes are not overlapping. The described method is illustrated in Figure 5 showing an example measurement analysis of the vibrating absorber at a perturbation frequency of $0.05 \mathrm{~Hz}$.

The ratio of the peak height value of the output signal in frequency domain to the peak height value of the input signal in frequency domain is the gain of the ZPTF for the given frequency.

The errors of each measurement point are just evaluated with the standard deviations of the fit functions. Failures of the data acquisition system, the absorber movement, and the determination of the reactivity influences via the compensation method are neglected, because these errors are an order of magnitude lower than the errors of the fit functions. An assessment of this assumption is shown in [9]. 
Table I. Simulated precursor parameters:

Precursor group fractions $\left(\boldsymbol{\beta}_{\boldsymbol{i}}\right)$, fraction of all precursor groups $\left(\boldsymbol{\beta}_{\text {eff }}\right)$ and corresponding lifetimes $\left(\boldsymbol{\lambda}_{\boldsymbol{i}}\right)$ with standard deviation (Std. dev.) derived from MCNP calculations.

\begin{tabular}{|c|c|c|c|c|}
\hline $\begin{array}{c}\text { Group of } \\
\text { precursor }\end{array}$ & $\beta_{i}$ & Std. dev. & $\begin{array}{c}\lambda_{i} \\
\text { in s }^{-1}\end{array}$ & $\begin{array}{c}\text { Std. dev. } \\
\text { in s }^{-1}\end{array}$ \\
\hline 1 & 0.00027 & 0.00001 & 0.01334 & 0.00001 \\
\hline 2 & 0.00137 & 0.00002 & 0.03273 & 0.00001 \\
\hline 3 & 0.00133 & 0.00002 & 0.12079 & 0.00001 \\
\hline 4 & 0.00296 & 0.00003 & 0.30293 & 0.00001 \\
\hline 5 & 0.00123 & 0.00002 & 0.85011 & 0.00001 \\
\hline 6 & 0.00050 & 0.00001 & 2.85508 & 0.00003 \\
\hline Sum & 0.00766 & 0.00004 & - & - \\
\hline
\end{tabular}

The reactor kinetic parameters were determined with an MCNP 6.0 [10] model of the AKR-2 and the ENDF/B-VIII.0 data library [11] for six groups of precursors. Where $l=57.2956 \cdot 10^{-6} \mathrm{~s}$ with a standard deviation of $8.50 \cdot 10^{-8} \mathrm{~s}$. The other parameters can be seen in Table I.

The measurements including the error-bars and the theoretical shape of the ZPTF derived by the simulation can be seen in Figure 6. The larger errors for the absorber of variable strength in comparison with the vibrating absorber result from the comparably small reactivity effect of the absorber of variable strength. In future measurements, using larger output signal lengths, can reduce the errors.

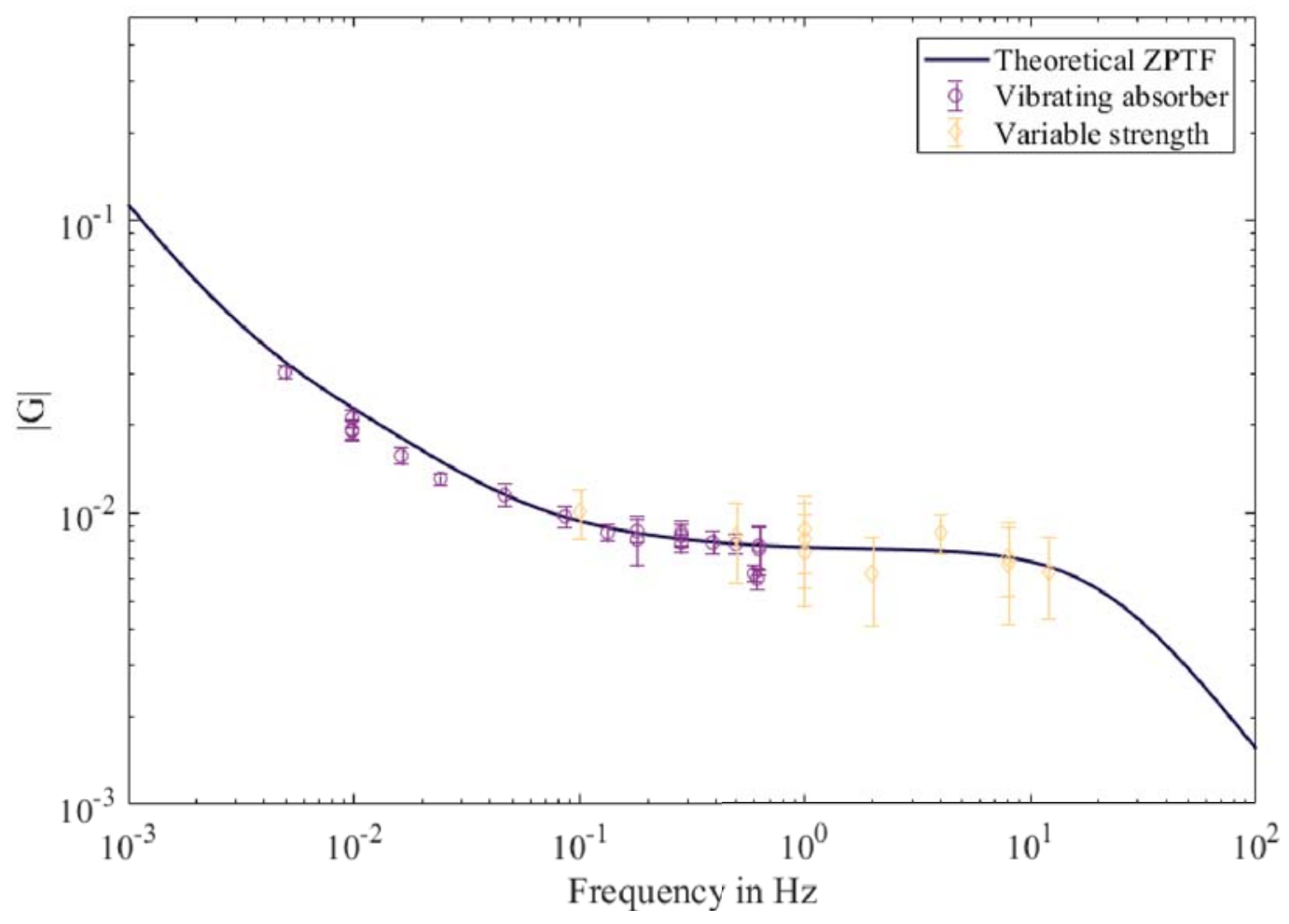

Figure 6. Comparison of the measurements and the theoretical shape of the ZPTF. 
Over all, a good agreement of the theoretical shape of the ZPTF and the measurement data can be stated. For a final proof of the used methods, it would be useful to extend the frequency range to frequencies up to $100 \mathrm{~Hz}$, to observe the reactor's behavior on frequencies higher than the upper bound of the plateau region of the ZPTF. It is also necessary to install a position encoder at the drive of the absorber of variable strength to reduce the error of the corresponding measurement points. This will also allow the investigation of the phase of the ZPTF, assuming a timely tied data acquisition of input and output signals.

\section{CONCLUSIONS}

The gain of the ZPTF of the AKR-2 was experimentally determined. Two absorbers of different kinds were used in overlapping frequency ranges. The measured data was analyzed with the help of discrete FFT. The obtained data was compared with the theoretical shape of the ZPTF, derived with the help of MCNP generated kinetic reactor parameters. The applicability of the used experimental setups could be shown within some limitations. For future experiments, a broadening of the used frequency range should be applied. A synchronized recording of the input and output signals would enable the evaluation of the phase of the ZPTF.

\section{ACKNOWLEDGMENTS}

The authors want to thank L. Beyer, M. Fussan and M. Krautz from the Institute of Complex Materials, Leibniz IFW Dresden for the CT of the detector. The research leading to these results has received funding from the Euratom research and training program 2014-2018 under grant agreement No 754316.

\section{REFERENCES}

1. D. L. Hetrick, Dynamics of Nuclear Reactors, Chapter 3, The University of Chicago Press, Chicago and London (1971).

2. I. Pázsit and C. Demazière, "Noise Techniques in Nuclear Systems," in Handbook of Nuclear Engineering, D. G. Cacuci, Ed. Boston, MA: Springer US, pp. $1629-1737$ (2010).

3. J.M. Harrer, R.E. Boyar and D. Krucoff, Measurements of CP-2 Reactor Transfer Function, Argonne National Laboratory, Chicago (1952).

4. C. Demazière, P. Vinai, M. Hursin, S. Kollias, and J. Herb, "Overview of the CORTEX project," in Proceedings of PHYSOR 2018, pp. 2971-2980 (2018).

5. R. Schröder, Zum kinetischen Verhalten des BER, Report, p. 8, Hahn-Meitner-Institut für Kernforschung, Berlin (1964).

6. B. Boehmer, M. Grantz, W. Hansen, D. Hinke, J. Konheiser, H.-C. Mehner, K. Noack, R. Schneider, I. Stephan and S. Unholzer, "Investigation of Gamma and Neutron Energy Fluences in Iron-Water Benchmark Configurations for the Verification of Monte Carlo Calculations and their Application in Reactor Material Dosimetry," J. Nucl. Sci. Technol., vol. 39, no. sup2, pp. 947-950 (2002).

7. P. S. Christensen, A Description of the Pile Oscillators at DR 1, Technical report, The Danish Atomic Energy Comission Research Establishment Risö (1966).

8. "Mathworks MatLab," https://de.mathworks.com/help/signal/ref/hamming.html (2019).

9. S. Hübner, Neuauslegung, Inbetriebnahme und Test eines Pile-Oszillators für den AKR-2, Thesis, TU Dresden, Dresden (2018).

10. T. Goorley, et al., "Initial MCNP6 Release Overview", Nuclear Technology, vol. 180, pp 298-315 (2012).

11. D.A. Brown et al., "ENDF/B-VIII.0: The 8th Major Release of the Nuclear Reaction Data Library with CIELO-project Cross Sections, New Standards and Thermal Scattering Data", Nuclear Data Sheets, vol. 148, pp. 1-142 (2018). 